\title{
Multidisciplinary decision-making in mitral valve disease: the mitral valve heart team
}

\author{
S. Heuts · J. R. Olsthoorn · S. M. M. Hermans · S. A. F. Streukens · J. Vainer · E. C. Cheriex · P. Segers · \\ J. G. Maessen · P. Sardari Nia
}

Published online: 11 February 2019

(C) The Author(s) 2019

\begin{abstract}
Background Although decision-making using the heart-team approach is apparently intuitive and has a class I recommendation in most recent guidelines, supportive data is still lacking. The current study aims to demonstrate the individualised clinical pathway for mitral valve disease patients and to evaluate the outcome of all patients referred to the dedicated mitral valve heart team.

Methods All patients who were evaluated for mitral valve pathology with or without concomitant cardiac disease between 1 January 2016 and 31 December 2016 were prospectively followed and included. Patients were evaluated, and a treatment strategy was determined by the dedicated mitral valve heart team. Results One hundred and fifty-eight patients were included; 67 patients were treated surgically (isolated and concomitant surgery), 20 by transcatheter interventions and 71 conservatively. Surgically treated patients had a higher 30 -day mortality rate $(4.4 \%)$, which decreased when specified to a dedicated surgeon $(1.7 \%)$ and in primary, elective cases $(0 \%)$. This was also observed for major adverse events within 30 days. Residual mitral regurgitation >grade 2 was more frequent in the catheter-based intervention group (23.5\%) compared to the surgical group (4.8\%).
\end{abstract}

\footnotetext{
S. Heuts $\cdot$ J. R. Olsthoorn $\cdot$ S. M. M. Hermans $\cdot$ P. Segers · J. G. Maessen $\cdot$ P. Sardari Nia $(\bowtie)$

Department of Cardiothoracic Surgery, Maastricht University Medical Centre, Maastricht, The Netherlands peyman.sardarinia@mumc.nl
}

S. Heuts · J. G. Maessen · P. Sardari Nia

Cardiovascular Research Institute Maastricht (CARIM), Maastricht University, Maastricht, The Netherlands

S. A. F. Streukens · J. Vainer · E. C. Cheriex Department of Cardiology, Maastricht University Medical Centre, Maastricht, The Netherlands
Conclusion In conclusion, the implementation of a multidisciplinary heart team for mitral valve disease is a valuable approach for the selection of patients for different treatment modalities. Our research group will focus on a future comparative study using historical cohorts to prove the potential superiority of the dedicated multidisciplinary heart-team approach.

Keywords Mitral valve disease - Heart team - Multidisciplinary $\cdot$ Decision-making

\section{Background}

The concept of a multidisciplinary decision-making team is well established in various medical disciplines $[1,2]$ and has been associated with improved survival $[3,4]$. Recently, such multidisciplinary teams have been introduced in the fields of cardiology and cardiac surgery, specifically to make decisions regarding coro-

What's new?

- Although the heart-team approach is intuitive, its implementation has not been described previously for mitral valve disease.

- To date, the conventional heart team has consisted of a random interventional cardiologist and surgeon, convening in a random composition without continuity. The mitral valve heart team consists of mitral experts convening weekly in the same composition.

- All patients referred for mitral valve disease undergo a standardised diagnostic pathway to facilitate an individualised approach.

- We observed a relatively high incidence of incidentalomas on computed tomography in the surgically treated group. 
nary revascularisation and transcatheter aortic valve replacement [5-7]. Although decision-making in the so-called heart team is apparently intuitive and has a class I recommendation in most recent guidelines [8-10], supporting comparative data is still lacking [11-13]. For mitral valve disease, only a few studies have reported first experience in multidisciplinary decision-making, limited to transcatheter mitral valve therapies $[14,15]$. The variety in mitral valve treatment options is increasing with transcatheter and offpump surgical interventions [16-18]. Furthermore, surgical mitral valve repair has proven to be associated with a steep learning curve, and outcome is significantly procedural volume related $[19,20]$. Therefore, a dedicated mitral valve care team seems even more warranted for treatment of mitral valve disease. Recently, we introduced the concept of a dedicated mitral valve heart team at our centre. This multidisciplinary approach focuses on a balanced treatment strategy for individual patients based on their specific mitral valve pathology, anatomical eligibility, comorbidities, background and wishes. The aim of the current study is to demonstrate standardised diagnostic pathways in mitral valve patients, give insight into our strategy of clinical decision-making for allocation of an individualised treatment pathway and to demonstrate the clinical outcome of all patients referred to the dedicated mitral valve heart team.

\section{Methods}

All consecutive patients whose mitral valve pathology was discussed by the dedicated mitral valve heart team between 1 January 2016 and 31 December 2016 were prospectively included in the current study. Patients were referred from four regional hospitals or by our own centre. Data were collected prospectively.

\section{The mitral valve heart team}

The traditional heart team consists of one cardiac surgeon and one interventional cardiologist with random subspecialties, and team members rotate frequently. Furthermore, patients are discussed by different heart teams during their work-up, implying a lack of continuity.

However, the mitral valve heart team consists of a dedicated mitral valve surgeon, one interventional cardiologist with experience in catheter-based mitral valve therapies and two imaging cardiologists with expertise in advanced echocardiography (one senior imaging cardiologist with $>30$ years of experience, 100-150 procedures annually; one fellow imaging cardiologist with 2 years of experience, 200 procedures annually, EACVI certified). Meetings of the mitral valve heart team were convened once a week and took place only if all members were present. All referred patients underwent transthoracic echocardiography at the site of referral, but all echocardiograms were evaluated by the heart team for severity and mechanism of mitral regurgitation (MR). When a patient was allocated to surgical treatment, valve reparability was assessed. Patient characteristics, valvular pathology and patient anatomy were considered and discussed comprehensively for treatment allocation. All degenerative valves were deemed eligible for repair. Isolated valve repairs/replacements were evaluated for an endoscopic approach.

In addition to diagnosis and determination of treatment strategy, the complete mitral valve heart team is also involved in the treatment phase, when interventions are evaluated by the dedicated imaging cardiologists in the operating room, and patients are evaluated and treated postoperatively by members of the team. Finally, in cases of late complications or recurrence of MR, patients are reintroduced to the mitral valve heart team for evaluation and indication for potential additional therapies.

\section{Mitral interventions}

At the Heart and Vascular Institute of our centre, a variety of mitral valve therapies are provided, divided in three groups: surgical, catheter-based interventions or conservative (pharmacological) treatment.

Surgical mitral valve repair or replacement is performed by means of sternotomy or fully endoscopically. In selected patients, mitral valve repair can be performed on the beating heart through a transapical approach (NeoChord, NeoChord Inc., Minneapolis, MN, USA) [16, 21]. Percutaneous treatments performed by the interventional cardiologist include edge-to-edge repair (MitraClip, Evalve Inc, Menlo Park, CA, USA) [18] and percutaneous annuloplasty (Carillon, Cardiac Dimension, Kirkland, WA, USA) [17].

\section{Diagnostic modalities}

All patients underwent transthoracic echocardiography (TTE) at the site of referral. Additionally, all patients eligible for surgical or transcatheter mitral valve repair underwent three-dimensional (3D) transoesophageal echocardiography (TOE) and all patients with isolated mitral valve pathology eligible for surgical intervention underwent computed tomography $(\mathrm{CT})$ for $3 \mathrm{D}$ anatomical reconstruction of the aorta and peripheral vessels to assess eligibility for an endoscopic approach [22]. Coronary angiography (CAG) was performed for evaluation of potential concomitant coronary artery disease.

\section{Outcomes}

Baseline risk assessment and clinical symptom severity was graded by the European System for Cardiac Operative Risk Evaluation (EuroSCORE) and New York Heart Association classification for dyspnoea 
respectively. Echocardiographic characteristics were assessed and quantified using an integrative approach [23].

Safety outcomes were defined as mortality and major adverse cardiac and cerebrovascular events (MACCE) within 30 days (mortality within 30 days, myocardial infarction, reoperation for failure of surgical repair, stroke, renal failure, deep wound infection, sepsis) and overall survival.

\section{Statistical analysis}

The distribution of continuous variables was assessed for normality using the Shapiro-Wilk test. Continuous variables are presented as mean \pm standard deviation or median and range in the presence of skewness. Categorical variables are presented as frequencies and percentages. Survival was estimated by the Kaplan-
Meier method. Data analysis was performed using commercially available software (SPSS version 24, IBM, Armonk, NY, USA).

\section{Results}

A total of 158 consecutive patients were discussed by the mitral valve heart team. Patients were allocated to their designated treatment modality. Sixty-seven patients were treated surgically, 20 with catheter-based interventions and 71 conservatively (Fig. 1).

The repair rate of MR based on degenerative disease was $100 \%$. Within the surgically treated group, 46 patients underwent on-pump surgical mitral valve repair, 8 beating heart mitral valve repairs were performed, and 13 patients underwent biological or mechanical mitral valve replacement for rheumatic or ischaemic disease or systolic anterior motion.

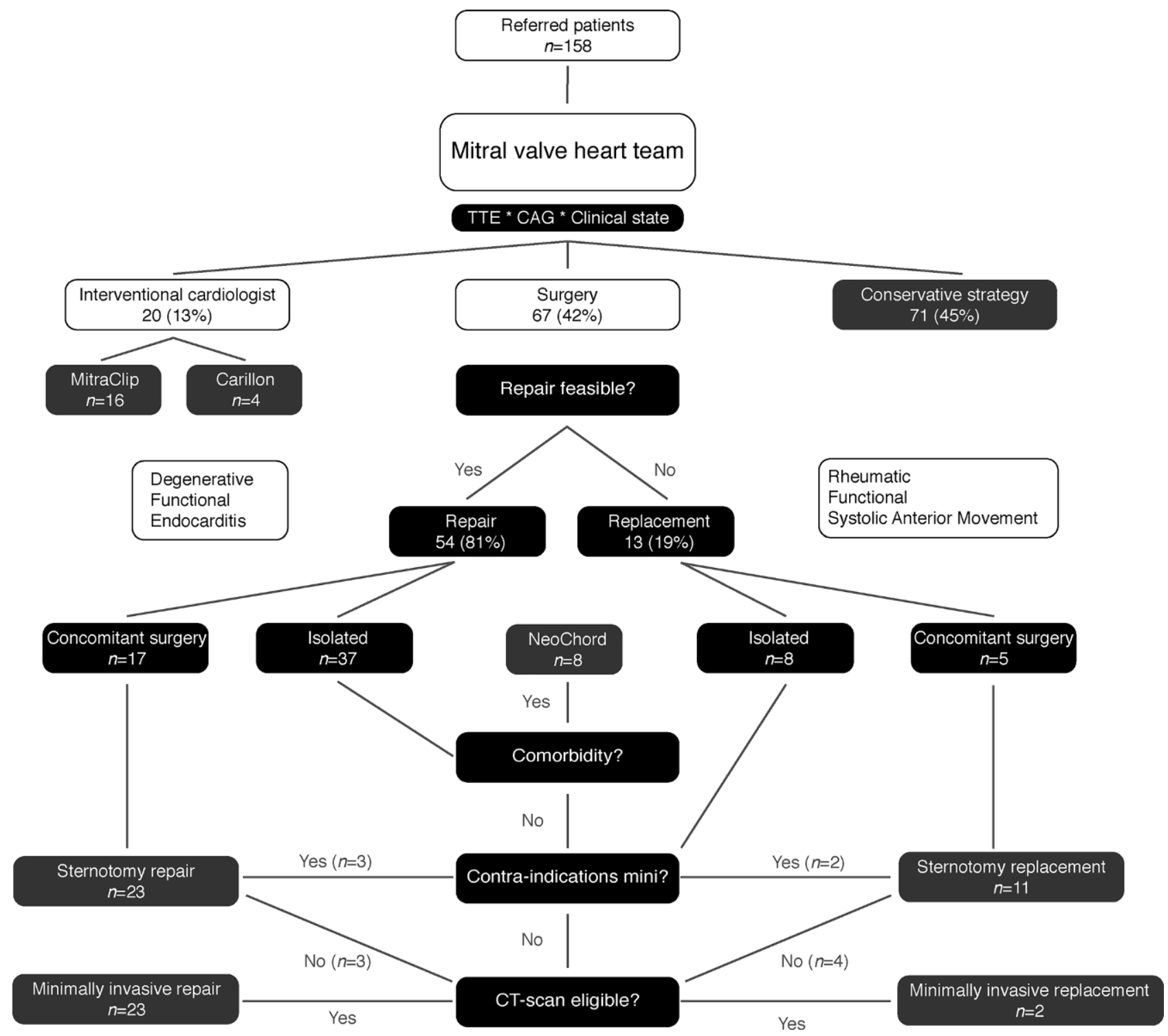

Fig. 1 Flowchart of decision-making in the mitral valve heart team. TTE transthoracic echocardiography, CAG coronary angiography, CT computed tomography 
Table 1 Baseline and surgical characteristics
Table 2 Baseline echocardiographic parameters

\begin{tabular}{|l|l|l|l|}
\hline & $\begin{array}{l}\text { Surgery } \\
(n=67)\end{array}$ & $\begin{array}{l}\text { Catheter-based interventions } \\
(n=20)\end{array}$ & $\begin{array}{l}\text { Conservative } \\
(n=71)\end{array}$ \\
\hline Age (years) & $63(15)$ & $69(11)$ & $73(11)$ \\
\hline Gender (male) & $43(64 \%)$ & $16(80 \%)$ & $35(49 \%)$ \\
\hline BMI (kg/m²) & $26.5[23.3-29.0]$ & $24.3[22.3-27.6]$ & $25.1[23.1-28.2]$ \\
\hline Diabetes & $8(11 \%)$ & $1(5 \%)$ & $10(14 \%)$ \\
\hline PHT & $30(45 \%)$ & $11(55 \%)$ & $34(48 \%)$ \\
\hline Reoperation & $3(5 \%)$ & $5(25 \%)$ & $16(23 \%)$ \\
\hline EuroSCORE log & $4.38[2.21-7.83]$ & $4.57[2.78-7.59]$ & $6.51[3.22-10.30]$ \\
\hline EuroSCORE II & $1.51[0.88-3.19]$ & $2.03[1.53-3.04]$ & $2.33[1.35-4.13]$ \\
\hline NYHA classification & & & $12(17 \%)$ \\
\hline No dyspnoea & $12(17 \%)$ & $2(10 \%)$ & $2(3 \%)$ \\
\hline I & $2(3 \%)$ & $1(5 \%)$ & $32(45 \%)$ \\
\hline II & $26(39 \%)$ & $11(55 \%)$ & $23(32 \%)$ \\
\hline III & $22(33 \%)$ & $6(30 \%)$ & $2(3 \%)$ \\
\hline IV & $5(8 \%)$ & 0 & \\
\hline $\begin{array}{l}\text { Surgery type } \\
\text { Isolated MVS }\end{array}$ & $45(67 \%)$ & & \\
\hline $\begin{array}{l}\text { Concomitant surgery } \\
\text { Surgical approach }\end{array}$ & $22(33 \%)$ & & \\
\hline $\begin{array}{l}\text { Endoscopic } \\
\text { (\% isolated valves) }\end{array}$ & $25(68 \%)$ & & \\
\hline $\begin{array}{l}\text { Sternotomy } \\
\text { (\% isolated valves) }\end{array}$ & $12(32 \%)$ & & \\
\hline $\begin{array}{l}\text { BMI body mass index, } \\
\text { NYHA New York Heart Association classification for }\end{array}$ & dyspnoea, MVS mitral valve surgery \\
\hline
\end{tabular}

\begin{tabular}{|c|c|c|c|}
\hline & $\begin{array}{l}\text { Surgery } \\
(n=67)\end{array}$ & $\begin{array}{l}\text { Catheter-based interventions } \\
(n=20)\end{array}$ & $\begin{array}{l}\text { Conservative } \\
(n=71)\end{array}$ \\
\hline LVEF (\%) & 60 [54-63] & 29 [16-44] & 51 [19-75] \\
\hline LVEDD (mm) & $56(8)$ & 64 (12) & $59(9)$ \\
\hline \multicolumn{4}{|l|}{ MR severity } \\
\hline Grade I & 0 & 0 & $8(11 \%)$ \\
\hline Grade II & $2(3 \%)$ & 0 & $26(37 \%)$ \\
\hline Grade III & $4(6 \%)$ & $3(15 \%)$ & $12(17 \%)$ \\
\hline Grade IV & $61(91 \%)$ & 17 (85\%) & $18(25 \%)$ \\
\hline MS & 0 & 0 & $7(10 \%)$ \\
\hline \multicolumn{4}{|l|}{ MR cause } \\
\hline Degenerative & $43(64 \%)$ & $4(20 \%)$ & $20(31 \%)$ \\
\hline Functional & $14(21 \%)$ & $16(80 \%)$ & 35 (55\%) \\
\hline Rheumatic & $6(9 \%)$ & 0 & $5(8 \%)$ \\
\hline Endocarditis & $2(3 \%)$ & 0 & 0 \\
\hline SAM & $2(3 \%)$ & 0 & $2(3 \%)$ \\
\hline Other & 0 & 0 & $2(3 \%)$ \\
\hline \multicolumn{4}{|c|}{$\begin{array}{l}\text { Leaflet prolapse } \\
\text { (\% surgical degenerative) }\end{array}$} \\
\hline PML & \multicolumn{3}{|l|}{$30(70 \%)$} \\
\hline AML & \multicolumn{3}{|l|}{$4(9 \%)$} \\
\hline Bileaflet & \multicolumn{3}{|l|}{$9(21 \%)$} \\
\hline
\end{tabular}

In the catheter-based intervention group, 15 patients $(75 \%)$ underwent a percutaneous edge-to-edge repair and 5 patients (25\%) percutaneous annulo- plasty. Reasons for conservative treatment were: MR not severe enough for intervention (30 cases, $42 \%$ ), patient's wish (17 cases, 24\%), deteriorated clini- 

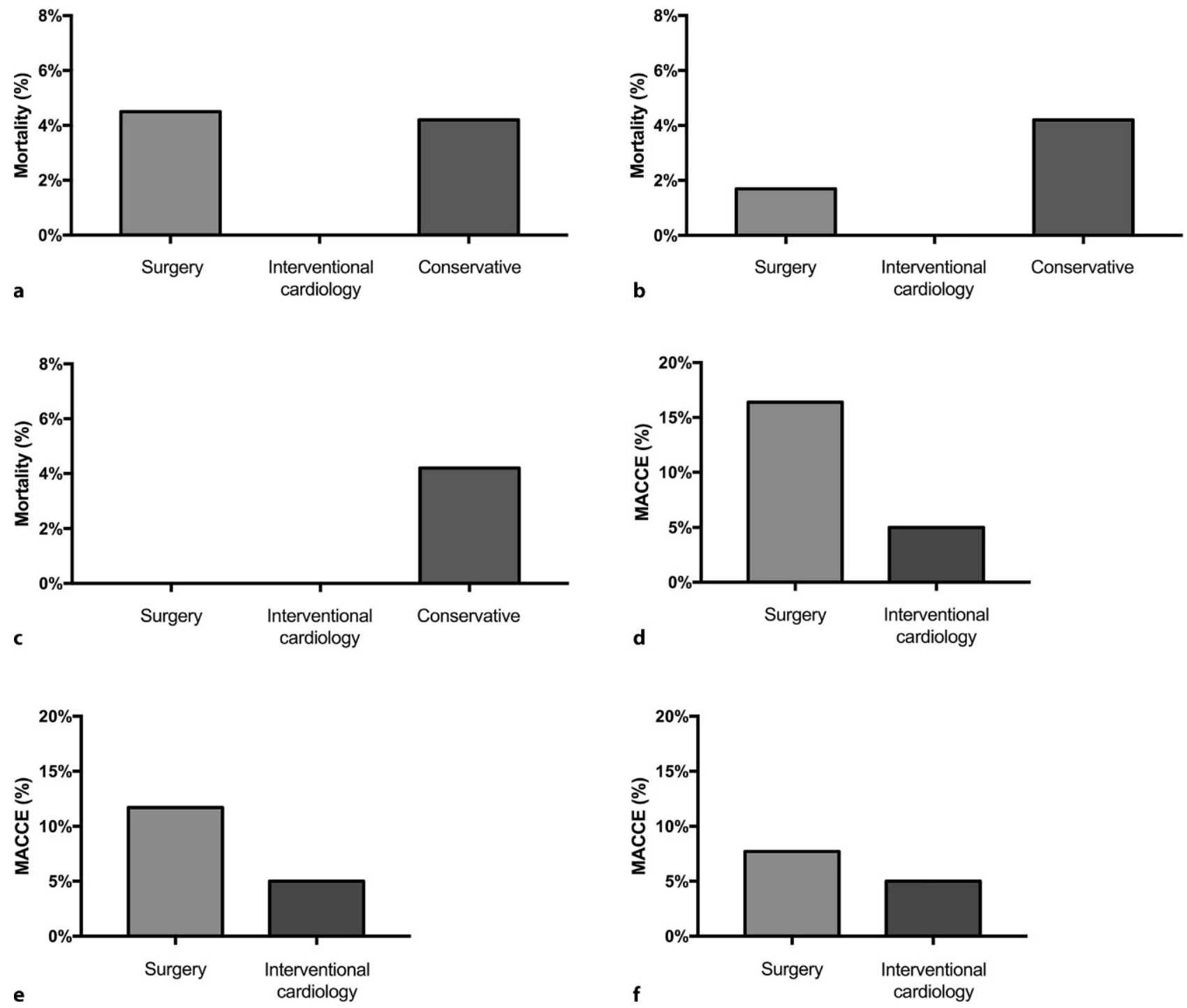

Fig. 2 Thirty-day mortality $(\mathbf{a}-\mathbf{c})$ rate and major adverse cardio- and cerebrovascular events (MACCE) (d-f) for the surgical, catheter-based intervention and conservative groups.

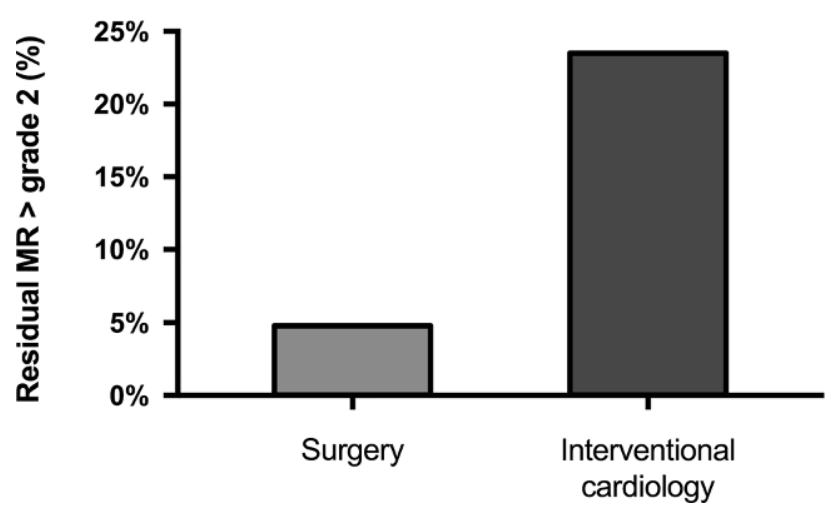

Fig. 3 Residual mitral regurgitation $(M R)>$ grade 2 for the surgical and catheter-based intervention group a, d Overall surgical group; b, e surgical group treated by a dedicated mitral valve surgeon; c, f primary, elective group treated by a dedicated mitral valve surgeon

cal state (16 cases, 23\%) and non-mitral surgical/ interventional treatment (8 cases, $11 \%$ ).

Baseline characteristics are depicted in Tab. 1. Surgically treated patients tended to be of younger age with fewer comorbidities and a lower surgical risk based on the EuroSCORE. Twenty-two patients (33\%) underwent concomitant surgery. An endoscopic approach was used in 23 of 35 patients with isolated valve disease, whereas sternotomy was performed in 12 patients. Reoperations, endocarditis and non-elective cases were included in the analyses as well. Baseline echocardiographic parameters are presented in Tab. 2.

Thirty-day mortality was assessed for all groups. There was no mortality within 30 days for the catheterbased intervention group, whereas 3 patients died within 30 days of decision-making in the conservative group $(4.2 \%)$. For surgically treated patients, 


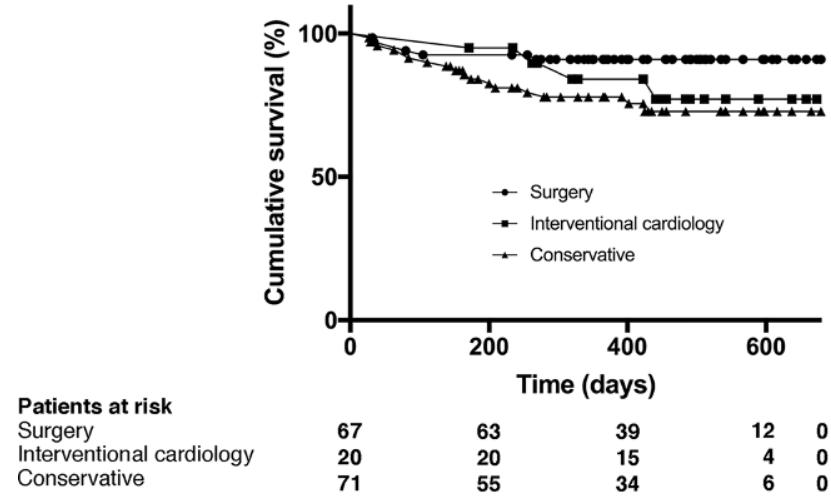

a

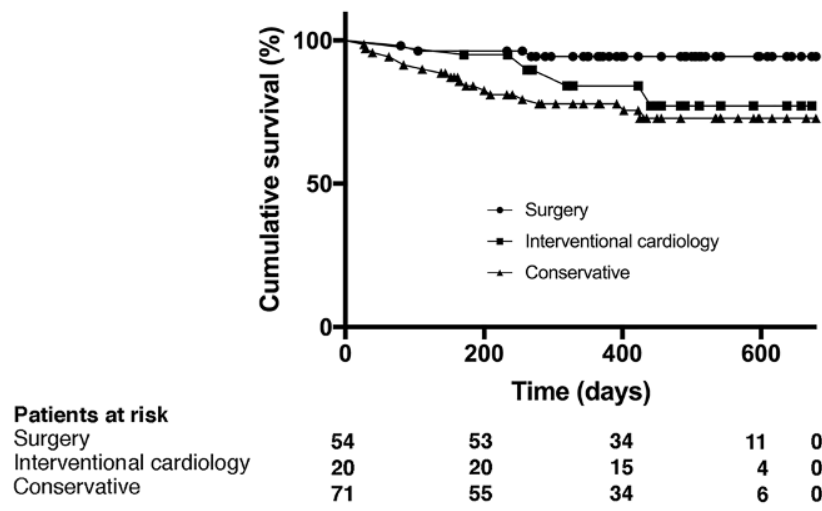

c

Fig. 4 a-d Survival analysis using Kaplan-Meier curves for the various treatments with a median follow-up of 450 days (range 138-673 days). a Overall surgical group, b surgical group treated by a dedicated mitral valve surgeon, c primary,

a distinction was made between (1) the overall group, (2) the group treated by a dedicated mitral valve surgeon, and (3) elective, primary cases operated on by a dedicated mitral valve surgeon. For the overall group ( $n=67$, treated by 3 surgeons) 30-day mortality was $4.4 \%$ ( 3 cases, Fig. $2 \mathrm{a}$ ). For the group treated by the dedicated surgeon $(n=60)$ 30-day mortality was $1.7 \%$ ( 1 case, a reoperation, Fig. $2 \mathrm{~b}$ ) and in the primary, elective group ( $n=57)$ no 30 -day mortality was observed (Fig. 2c).

A similar decrease in occurrence of MACCE within 30 days was found. In the catheter-based intervention group, 1 patient had to undergo a left ventricular assist device implant after edge-to-edge repair (5\%). Sixteen

Table 3 Incidentalomas

\begin{tabular}{l|l|}
\hline CT scans during work-up $(n=44)$ & $\begin{array}{l}\text { Incidentalomas } \\
(n=12,27 \%)\end{array}$ \\
\hline Abdominal mass/tumour & 4 \\
\hline Thoracic mass/tumour & 7 \\
\hline Abdominal aortic aneurysm & 1 \\
\hline Actual carcinomas & 4 \\
\hline$C T$ computed tomography &
\end{tabular}

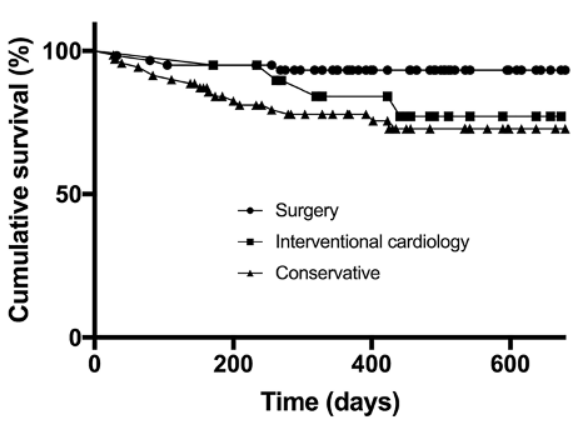

Patients at risk

Surgery

Interventional cardiology Conservative

$\begin{array}{rrrrr}60 & 58 & 36 & 11 & 0 \\ 20 & 20 & 15 & 4 & 0 \\ 71 & 55 & 34 & 6 & 0\end{array}$

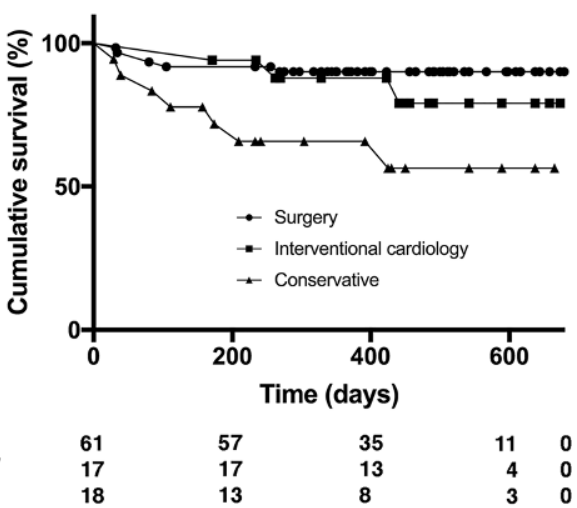

Patients at risk

Surgery
Interventional cardiology Conservative

d

elective group treated by a dedicated mitral valve surgeon, d stratified for patients with severe mitral regurgitation (MR). Numbers of patients at risk at a given time are given below each graph

percent of the patients in the surgically treated group had complications (Fig. 2d), 11.7\% in the dedicated group (Fig. 2e) and $7.7 \%$ in the primary, elective group (Fig. 2f).

Postoperative echocardiography performed at 3 months after discharge revealed $23.5 \%$ of patients treated with a catheter-based intervention to have residual $M R>$ grade 2 , compared to $4.5 \%$ in the surgically treated group (3 patients, Fig. 3 ).

Survival was estimated using the Kaplan-Meier method at a median follow-up of 450 days (range 138-673 days) and is depicted in Fig. 4 for the various groups, demonstrating beneficial long-term survival for surgically treated patients. In addition to the stratification for surgically treated groups, Fig. $4 \mathrm{~d}$ provides information on survival of the patient group with severe MR, revealing a poor short-term prognosis for the conservatively treated group.

\section{Additional observations}

All patients with isolated valve disease and without contraindications underwent contrast-enhanced CT angiography with $3 \mathrm{D}$ reconstruction to assess 
anatomical eligibility for endoscopic surgery $(n=44)$. Seven patients were excluded from an endoscopic approach based on CT due to suboptimal accessibility of the peripheral vessels for cardiopulmonary bypass cannulation or aberrant aortic diameters.

Furthermore, on these 44 scans, 12 incidentalomas were found, of which 4 were actual carcinomas requiring further follow-up and/or treatment (2 non-small cell lung carcinomas, 1 cholangiocarcinoma, 1 thyroid carcinoma) (Tab. 3).

\section{Discussion}

Although intuitive, there is no supportive data for the use of the heart team in decision-making for cardiovascular disease [11].

In this first study on a dedicated mitral valve heart team, we present the implementation of a recently introduced dedicated mitral valve heart team at our centre. The current study included all patients referred to our centre for mitral valve disease within 1 year (2016, $n=158$ ). Almost half of patients $(45 \%)$ were treated conservatively. This can be explained by (1) the high-risk population of patients with mitral stenosis and end-stage heart-failure, (2) the advanced age ( $>80$ years) and high-rate of severe pulmonary hypertension and (3) the fact that the mitral valve team is well established and known to referring centres, and patients are referred at an early stage of mitral valve disease. The early referral is illustrated by the number of patients who were treated conservatively due to an insufficient grade of MR for intervention (42\%). These patients will be followed up annually for disease progress. Twelve percent of patients were treated with a catheter-based intervention, using either percutaneous edge-to-edge repair $(75 \%)$ or percutaneous annuloplasty (25\%). These patients were older, with a higher surgical risk, but more importantly had a predominantly reduced left ventricular function. Therefore, a transcatheter intervention was indicated.

Sixty-seven patients $(43 \%)$ were treated surgically. Mitral valve reparability was assessed by the heart team preoperatively, and a repair rate of $100 \%$ was achieved for degenerative valves. Of all mitral pathologies, $84 \%$ were repaired, while $16 \%$ were replaced. Of note is the fact that isolated and concomitant mitral surgery were both included in the analysis. In selected cases, transapical beating heart valve repair (NeoChord) was performed when patients were deemed eligible. These older patients, with an overall elevated surgical risk and comorbidities, were eligible for repair but were expected to have a complicated postoperative course.

Furthermore, with the emergence of several multimodality imaging techniques, the current study provides an algorithm for the use of the modalities (CT, $3 \mathrm{D}$ anatomical reconstructions, TTE, TOE, CAG) in various stages of the standardised diagnostic pathway. This algorithm, provided in Fig. 1, could prevent unnecessary diagnostics and reduce associated costs, patient burden and exposure to radiation.

A routine CT thorax scan was performed in patients being evaluated for endoscopic mitral valve surgery. We were able to exclude 7 patients in the preoperative course because of inaccessibility or unsuitability of the vessels for this approach. Eventually 25 patients underwent endoscopic surgery, in which no conversions occurred.

Catheter-based interventions proved to be safe (no 30-day mortality, 5\% MACCE), but had a relatively high probability of residual MR > grade 2 (23.5\%) compared to the surgical group $(4.5 \%)$, in line with prior studies [24]. Furthermore, after an initial uneventful course, these patients had shorter overall survival, presumably based on their age, poorer clinical state and diminished cardiac function.

A trend towards lower 30-day mortality with fewer major complications for patients treated by a dedicated surgeon was observed, confirming previous studies [25]. These studies demonstrated better outcomes and survival in mitral valve surgery when performed by a dedicated surgeon on a weekly basis after completion of the learning curve [19, 20, 26], indicating surgical volume to be a determinant of repair rate, freedom of reoperation and survival.

Survival was estimated with a median follow-up of 450 days. A relatively high cumulative mortality was observed in the conservatively treated group (25.4\%). Most deaths occurred in the subgroup which was treated conservatively because of a deteriorated clinical state. This finding was also observed in a comparable revascularisation study [27]. Additionally, conservatively treated patients with severe MR had a poor short-term prognosis, potentially explained by a combination of a myriad of factors contributing to a higher surgical risk, such as advanced age, severe mitral stenosis with subsequent end-stage heart-failure and a high rate of severe pulmonary hypertension.

As an additional observation, we found a relatively high rate of incidentalomas on preoperative CT scans performed in the planning of endoscopic surgery. Out of 44 patients, 12 incidentalomas (27\%) were found, of which 4 (9\%) were actual carcinomas requiring further follow-up and/or treatment. Several other CT screening studies describe a lower prevalence of tumours on screening [28]. However, little is known yet about the complex interplay between cardiovascular disease and cancer, which could both be a different manifestation of common underlying risk factors [29], explaining this finding in a patient group with extensive cardiovascular disease.

\section{Limitations}

The current study cohort consists of a relatively small heterogeneous group $(n=158)$. The study represents a single-arm study in which the superiority of the multidisciplinary heart-team approach cannot be proven. 
However, this was beyond our scope, as we aimed to demonstrate the prospective results of implementation of a dedicated mitral valve heart team in a centre performing a broad range of mitral valve therapies. Furthermore, the study is subject to selection bias for the described treatment modalities and is therefore not able to detect potential differences between these therapies. However, the current study is the first to describe and give insight into clinical decision-making in a mitral valve disease patient group as a whole and will serve as a scientific basis for future studies on a multidisciplinary approach, in order eventually to potentially prove its superiority.

As it seems unethical to study the heart team in a randomised fashion, our research group is focussing on a future study, using a historical cohort, in order to provide potential evidence for superiority of the dedicated heart-team approach.

\section{Conclusion}

The current study demonstrated the implementation of a multidisciplinary mitral valve heart team, gave insight into our strategy for clinical decision-making and treatment allocation, and demonstrated shortterm clinical outcomes of patients with mitral valve disease. Our research group will focus on a comparative study with historical cohorts, potentially providing a scientific basis for the current recommendations in guidelines, as we believe a multidisciplinary approach will improve efficiency and patient outcome.

Conflict of interest S. Heuts, J.R. Olsthoorn, S.M.M. Hermans, S.A.F. Streukens, J. Vainer, E.C. Cheriex, P. Segers, J.G. Maessen and P. Sardari Nia declare that they have no competing interests.

Open Access This article is distributed under the terms of the Creative Commons Attribution 4.0 International License (http://creativecommons.org/licenses/by/4.0/), which permits unrestricted use, distribution, and reproduction in any medium, provided you give appropriate credit to the original author(s) and the source, provide a link to the Creative Commons license, and indicate if changes were made.

\section{References}

1. Mendelssohn DC. Coping with the CKD epidemic: the promise of multidisciplinary team-based care. Nephrol Dial Transplant. 2005;20:10-2.

2. Junor EJ, Hole DJ, Gillis CR. Management of ovarian cancer: referral to a multidisciplinary team matters. Br J Cancer. 1994;70:363-70.

3. Forrest LM, McMillan DC, McArdle CS, et al. An evaluation of the impact of a multidisciplinary team, in a single centre, on treatment and survival in patients with inoperable nonsmall-cell lung cancer. Br J Cancer. 2005;93:977-8.

4. Kesson EM, Allardice GM, George WD, et al. Effects of multidisciplinary team working on breast cancer survival: retrospective, comparative, interventional cohort study of 13722 women. BMJ. 2012;344:e2718.
5. Sintek M, Zajarias A. Patient evaluation and selection for transcatheter aortic valve replacement: the heart team approach. Prog Cardiovasc Dis. 2014;56:572-82.

6. Holmes DR Jr., Mohr F, Hamm CW, et al. Venn diagrams in cardiovascular disease: the Heart Team concept. Eur Heart J. 2014;35:66-8.

7. Denvir MA, Pell JP, Lee AJ, et al. Variations in clinical decision-making between cardiologists and cardiac surgeons; a case for management by multidisciplinary teams? JCardiothorac Surg. 2006;1:2.

8. Kolh P, Windecker S. ESC/EACTS myocardial revascularization guidelines 2014. Eur Heart J. 2014;35:3235-6.

9. Baumgartner H, Falk V, Bax JJ, et al. 2017 ESC/EACTS Guidelines for the management of valvular heart disease. Eur HeartJ. 2017;38:2739-91.

10. Nishimura RA, Otto CM, Bonow RO, et al. 2014 AHA/ACC guideline for the management of patients with valvular heart disease: a report of the American College of Cardiology/American Heart Association Task Force on Practice Guidelines. JThorac Cardiovasc Surg. 2014;148:e1-e132.

11. Coylewright M, Mack MJ, Holmes DR Jr., et al. A call for an evidence-based approach to the Heart Team for patients with severe aortic stenosis. J Am Coll Cardiol. 2015;65:1472-80.

12. Holmes DR Jr., Rich JB, Zoghbi WA, et al. The heart team of cardiovascular care. JAm Coll Cardiol. 2013;61:903-7.

13. RosenscheinU,NaglerRM, RofeA.Theheartteamapproach to coronary revascularization-have we crossed the lines of evidence-based medicine? Am J Cardiol. 2013;112:1516-9.

14. Treede H, Schirmer J, Rudolph V, et al. A heart team's perspective on interventional mitral valve repair: percutaneous clip implantation as an important adjunct to a surgical mitral valve program for treatment of high-risk patients. J Thorac Cardiovasc Surg. 2012;143:78-84.

15. Baldus S, Schillinger W, Franzen O, et al. MitraClip therapy in daily clinical practice: initial results from the German transcatheter mitral valve interventions (TRAMI) registry. Eur J Heart Fail. 2012;14:1050-5.

16. SeeburgerJ, Rinaldi M, Nielsen SL, etal. Off-pumptransapical implantation of artificial neo-chordae to correct mitral regurgitation: the TACT Trial (Transapical Artificial Chordae Tendinae) proof of concept. J Am Coll Cardiol. 2014;63:914-9.

17. Schofer J, Siminiak T, Haude M, et al. Percutaneous mitral annuloplasty for functional mitral regurgitation: results of the CARILLON Mitral Annuloplasty Device European Union Study. Circulation. 2009;120:326-33.

18. Feldman T, Foster E, Glower DD, et al. Percutaneous repair or surgery for mitral regurgitation. N Engl J Med. 2011;364:1395-406.

19. Holzhey DM, Seeburger J, Misfeld M, et al. Learning minimally invasive mitral valve surgery: a cumulative sum sequential probability analysis of 3895 operations from a single high-volume center. Circulation. 2013;128:483-91.

20. Bolling SF, Li S, O’Brien SM, et al. Predictors of mitral valve repair: clinical and surgeon factors. Ann Thorac Surg. 2010;90:1904-11. discussion 12.

21. Heuts S, Streukens S, Daemen J, et al. Preoperative planning of transapical beating heart mitral valve repair for safe adaptation in clinical practice. Innovations (Phila). 2018;13(3):200-6.

22. Heuts S, Maessen JG, Sardari NP. Preoperative planning of left-sided valve surgery with 3D computed tomography reconstruction models: sternotomy or a minimally invasive approach? Interact Cardiovasc Thorac Surg. 2016;22:587-93. 
23. Lancellotti P, Moura L, Pierard LA, et al. European Association of Echocardiography recommendations for the assessment of valvular regurgitation. Part 2: mitral and tricuspid regurgitation (native valve disease). Eur J Echocardiogr. 2010;11:307-32.

24. Alozie A, Paranskaya L, Westphal B, et al. Clinical outcomes of conventional surgery versus MitraClip(R) therapy for moderate to severe symptomatic mitral valve regurgitation in the elderly population: an institutional experience. BMC Cardiovasc Disord. 2017;17:85.

25. Gammie JS, Sheng S, Griffith BP, et al. Trends in mitral valve surgery in the United States: results from the Society of Thoracic Surgeons Adult Cardiac Surgery Database. Ann Thorac Surg. 2009;87:1431-7. discussion 7-9.
26. Chikwe J, Toyoda N, Anyanwu AC, et al. Relation of mitral valve surgery volume to repair rate, durability, and survival. J Am Coll Cardiol. 2017;69(19):2407-9.

27. Chu D, Anastacio MM, Mulukutla SR, et al. Safety and efficacy of implementing a multidisciplinary heart team approach for revascularization in patients with complex coronary artery disease: an observational cohort pilot study. JAMA Surg. 2014;149:1109-12.

28. Pedersen JH, Ashraf H, Dirksen A, et al. The Danish randomized lung cancer $\mathrm{CT}$ screening trial-overall design and results of the prevalence round. J Thorac Oncol. 2009;4:608-14.

29. Koene RJ, Prizment AE, Blaes A, et al. Shared risk factors in cardiovascular disease and cancer. Circulation. 2016;133:1104-14. 